m09.004

\section{Small molecules with big impact: fine static and dynamic detail in the atomic resolution structures of hydroxynitril lyase from Hevea brasiliensis give insight into mechanisms of ligand binding}

\author{
Andrea Schmidt $^{\mathrm{a}}$, Christoph Kratky ${ }^{\mathrm{b}}$, Victor S. Lamzin ${ }^{\mathrm{a}}$ \\ ${ }^{a}$ European Molecular Biology Laboratory, c/o DESY, Notkestrasse 85, \\ D-22603 Hamburg. ${ }^{b}$ Institut für Chemie, Karl-Franzens Universität Graz, \\ Heinrichstrasse 28, A-8010 Graz
}

\section{Keywords: atomic resolution, anisotropic atomic displacement parameters, catalysis, protonation state}

Hydroxynitrile lyase (HNL) from Hevea brasiliensis is an enzyme of industrial use for the enantioselective production of cyanohydrins for organic synthesis from HCN and ketones.

Previous X-ray crystallographic studies have helped to elucidate its reaction mechanism of HNL, yet some questions still remained unanswered. Although the catalytic reaction is base catalysed, the enzyme's $\mathrm{pH}$ optimum lies within the $\mathrm{pH}$ region of $5-6$. The pKa of the active site residues, in particular of those constituting the catalytic triad, is apparently adaptable subject to the conditions and the nature of the incoming ligands. Furthermore, the protonation state of adjacent residues relevant for ligand binding is yet unclear. Therefore, the effects of the ligands as well as the influence of the solvent $\mathrm{pH}$ on the protonation state in the active centre are the focus of the present study.

The access to the HNL active site is very restricted; thus certain conformational changes must occur in order to admit the substrates and to release the products. We have developed a software tool that allows extracting directional motion of atoms, residues or groups of residues directly from the anisotropic atomic displacement parameters. In addition to various visualisation tools, the data include comprehensive statistics on the anisotropic behaviour within the protein and the assignment of regions of concerted motion.

We collected a number of atomic resolution X-ray datasets on $\mathrm{HNL}$ in the native state as well as with soaked ligands such as acetone (one of the educts or products), isopropanol and thiocyanate. The structures were refined over the full resolution range including hydrogen atoms and anisotropic ADPs. The four crystal structures revealed the binding modes of the substrate analogues, which allowed the determination of the relative positions of the two co-substrates prior to cyanohydrin synthesis as well as monitoring changes in the arrangement and protonation state in the active site. Analysis of the anisotropic ADPs revealed differences in directional motion of the residues in the active site. In the cap region covering the active site the primary direction of motion changes relative to the hydrolase domain. The impact of ligand binding on the overall molecular motion as well as their correlation to fine structural details will be discussed.

[1] Wagner, U. G., Hasslacher, M., Griengl, H., Schwab, H. and Kratky, C. (1996) Structure 4, 811-822

[2] Gruber, K., Gugganig, M., Wagner, U. G. and Kratky, C. (1999) Biol. Chem. 380, 993-1000.

[3] Wagner, U. G., Schall, M., Hasslacher, M., Hayn, M., Griengl, H., Schwab, H. and Kratky, C. (1996) Acta Cryst. D52, 591-593. m09.005

\section{Reaction mechanism of CCH type $\beta$-ketoacyl-ACP synthases}

Anette Henriksen $^{\mathrm{a}^{*}}$, Johan G. Olsen ${ }^{\mathrm{a}}$, Penny von Wettstein-Knowles ${ }^{\mathrm{b}}$

${ }^{a}$ Biostructure Group, Carlsberg Laboratory,DK2500-Valby, Denmark. ${ }^{b}$ Genetics Department, Molecular Biology and Physiology Institute, Copenhagen University, DK-1353 Copenhagen, Denmark. "E-mail: anette@crc.dk

\section{Keywords: fatty acid biosynthesis, reaction mechanism, active site structure}

$\beta$-ketoacyl-ACP synthase enzymes construct fatty acyl chains by adding $\mathrm{C}_{2}$ carbon units to the growing acyl chain in a three step Claisen condensation reaction. In this reaction the acyl primer is first transferred to the enzyme in a trans thioesterification from the acyl carrier protein. Next, a malonyl-acyl carrier protein is decarboxylated to form a carbanion intermediate, which in the final step attacks $\mathrm{C} 1$ of the primer substrate whereby the acyl chain is elongated by the $\mathrm{C}_{2}$ unit. $\beta$-ketoacyl-ACP synthases from mitochondria, some isozymes from plastids and bacteria and a domain of human fatty acid synthase, have a cysteine-histidine-histidine triad and a conserved lysine in the active site not observed in $\beta$-ketoacyl-ACP synthases with a cysteine-histidine-asparagine triad [1]. Crystal structures of wild type and eight mutant enzymes with and/or without bound substrate were determined to test the role of these active site residues [2]. That the $\mathrm{H} 298 \mathrm{E}$ enzyme has residual decarboxylase activity in the $\mathrm{pH}$ range 6-8, while the $\mathrm{H} 298 \mathrm{Q}$ enzyme does not catalyze decarboxylation at all shows that $\mathrm{H} 298$ serves as a catalytic base in the decarboxylation step. H333 is critical for decarboxylation activity. Mutagenesis of lysine 328 reveals that this residue plays a dual role in catalysis; its charge influences acyl transfer to the active site cysteine, while the steric restraint imposed on $\mathrm{H} 333$ makes $\mathrm{H} 333$ an obligate hydrogen bond donor at $\mathrm{N}_{\epsilon}$, directed only towards the active site and malonyl-ACP binding area in the fatty acid complex.

[1] Olsen, J.G., Kadziola, A., von Wettstein-Knowles, P., SiggaardAndersen, M. \& Larsen, S., Structures of $\beta$-ketoacyl-acyl carrier protein synthase I complexed with fatty acids elucidate its catalytic machinery. Structure, 2001, 9, 233.

[2] von Wettstein-Knowles, P., Olsen J.G., McGuire, K.A. \& Henriksen, A., Fatty acid synthesis. FEBS Letters, 2006, 273, 695. 